\title{
Reconocimiento de Expresiones Faciales Emocionales. Diferencias en el Desarrollo*
}

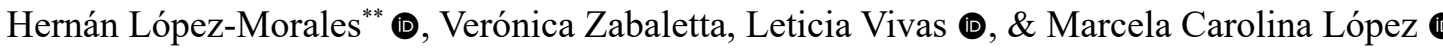 \\ Instituto de Psicología Básica, Aplicada y Tecnología, Universidad Nacional de Mar del Plata, \\ CONICET, Mar del Plata, Argentina
}

RESUMEN - El trabajo se propuso caracterizar el reconocimiento facial de emociones en población infanto-juvenil. Se administró una adaptación digital del Test Pictures of Facial Affects a 147 participantes de entre 9 y 18 años. Los resultados evidenciaron una asociación negativa entre la edad y la tasa de aciertos para alegría y positiva para asco y miedo. Además, se evidenció un efecto significativo de la edad en los tiempos de respuesta de todas las emociones a excepción del miedo. Los resultados sugieren que a medida que aumenta la edad el reconocimiento emocional es más veloz, sin embargo, esto se refleja en una mejoría en el reconocimiento emocional sólo en asco y miedo. Se discuten la importancia de estas emociones para la adolescencia.

PALABRAS CLAVE: reconocimiento de emociones faciales, cognición social, psicología del desarrollo

\section{Developmental Differences in the Recognition of Facial Expressions of Emotions}

\begin{abstract}
This research aimed to characterize the recognition of basic emotions in facial expressions in children and adolescents. A digital adaptation of the Pictures of Facial Affects test was administered to 147 participants of both sexes, between 9 and 18 years old. Results showed a negative association between age and rate of success in recognition of joy, and a positive association between age and rate of success in recognition of disgust and fear. A significant effect of age on the recognition of all emotions except fear was found regarding reaction times. These results suggest that as age increases, emotional recognition is faster. However, this is not reflected in an improvement in emotional recognition. The only cases in which recognition was enhanced with age were disgust and fear. The importance of these emotions for adolescence is discussed.
\end{abstract}

KEYWORDS: facial emotions recognition, social cognition, developmental psychology

\section{Reconhecimento de Expressões Faciais Emocionais: Diferenças no Desenvolvimento}

\begin{abstract}
RESUMO - Este trabalho se propôs a caracterizar o reconhecimento facial de emoções na população infanto-juvenil. Aplicou-se uma versão digital do Test Pictures of Facial Affects a 147 participantes com idades entre 9 e 18 anos. Os resultados evidenciaram uma associação negativa entre a idade e taxa de acertos para alegria, bem como uma associação negativa para nojo e medo. Identificou-se, ainda, um efeito significativo da idade nos tempos de resposta para todas as emoções, com exceção de medo. Os resultados sugerem que, conforme aumenta a idade, o reconhecimento facial se torna mais rápido. No entanto, isso só se refletiu em uma melhora no reconhecimento facial para nojo e medo. Discute-se a importância dessas emoções para a adolescência.
\end{abstract}

PALAVRAS-CHAVE: reconhecimento de expressões faciais, cognição social, psicología do desenvolvimento

\footnotetext{
* Apoio: Instituto de Psicología Básica, Aplicada y Tecnología (CONICET - UNMdP)

** E-mail: hernanlopezmorales@gmail.com

n Submetido: 07/11/2017; Revisado: 08/05/2018; Aceito: 04/07/2018.
} 
La neurociencia social (NCS) vincula la investigación en psicología social y las neurociencias cognitivas, asumiendo como objetivo el estudio de las bases biológicas de la cognición y las conductas sociales (Grande-García, 2009; Liberman, 2010). La base fundacional de toda esta empresa científica es la hipótesis de que las cogniciones sobre el entorno social son cualitativamente distintas e involucran diferentes estructuras cerebrales que aquellas que se realizan sobre cuestiones no sociales. En la última década, esta presunción ha recibido muchos aportes a su favor y, a su vez, tiene un fundamento evolutivo en la hipótesis del cerebro social. La misma enuncia que el tamaño del cerebro de los primates -comparativamente más grande que el de otros vertebrados- se debe a la necesidad de integrar grupos sociales inusualmente grandes y a la complejidad en el nivel de computación cerebral que esto acarrea (Dunbar, 2009; Singer, 2012). Una de las principales líneas de investigación dentro de esta disciplina es la que involucra las habilidades comprendidas en la cognición social, definida como procesos neurobiológicos, psicológicos y sociales, por medio de los cuales se perciben, reconocen y evalúan los eventos sociales, para construir representaciones de la relación entre uno mismo y los otros; empleando esas representaciones para guiar el comportamiento social (Adolphs, 2010). Así, la cognición social es un constructo complejo que implica varias habilidades distintas que, en conjunto, configuran el objeto de estudio de las neurociencias cognitivas sociales. $\mathrm{Si}$ bien los autores no coinciden en cuáles son específicamente esas habilidades, aquellas que han recibido más consenso son el reconocimiento o percepción de la emoción a través de estímulos faciales, percepción social, teoría de la mente, empatía, estilo de atribución y autoconciencia (Ruiz-Ruiz et al., 2006).

En este marco, el reconocimiento de emociones faciales es entendido como el proceso por el cual se perciben y analizan los rasgos faciales para la identificación del estado emocional que los mismos denotan (Adolphs, 2002). El mismo aporta información adicional para la interpretación de los mensajes y las acciones de los demás, desempeñando un papel central en la regulación de la conducta social como componente de la interacción interpersonal (GarcíaRodríguez et al., 2008). Esta línea de investigación estuvo principalmente orientada al estudio de las expresiones faciales de las emociones básicas, entendiendo a estas como estados somáticos ligados a conductas fundamentales para la supervivencia, en tanto acciones que se expresan en el rostro, la voz o en conductas específicas tendientes a mantener la homeostasis (Damasio, 2005; Miguel, 2015). La evaluación de las expresiones faciales para el estudio de las emociones ha sido el método más utilizado debido a que ofrece varias ventajas: (1) es el tipo de investigación más antiguo y el que ofrece un mayor cúmulo de conocimientos; (2) la noción de universalidad de las emociones ofrece a todos los estudiosos del tema una base conceptual para el entendimiento de las diferencias y similitudes culturales de la expresión facial;
(3) a través de la expresión facial es posible mostrar estados emocionales específicos, aportando información concreta tanto en el área motivacional como en la de la comunicación.

A mediados de la década de los ' 60 los trabajos de Paul Ekman reunieron evidencia suficiente para demostrar la existencia de seis expresiones universales de emoción: enojo, asco, miedo, alegría, tristeza y sorpresa (Ekman, 1994), siendo alguno de sus rasgos distintivos: fisiología particular, evaluación automática, presencia en otros primates, inicio rápido, breve duración $\mathrm{y}$, sobre todo, características faciales bien definidas (Ekman, 1999).

Durante la década del ' 90 , surgieron varias controversias en torno a esta teoría. Russell (1994), fue uno de sus mayores críticos, al encontrar diferencias en el reconocimiento de las expresiones dependiendo de la cultura de la que provenían los examinados. Sin embargo, para Matsumoto (2001), las discrepancias en los grados de reconocimiento emocional son debidas a que diferentes culturas poseen diferentes normas para el reconocimiento emocional; estas reglas de manifestación cultural (Ekman \& Friesen, 1969) son aprendidas desde temprana edad y dictan las pautas para el manejo y modificación de las expresiones universales dependiendo del contexto social (Ekman, 1972). Lejos de interpretar estas diferencias como evidencia en contra de la universalidad, estas diferencias enfatizan la importancia de las llamadas reglas de manifestación cultural que varían de cultura en cultura, enfatizando, enmascarando u ocultando ciertos patrones de expresión facial.

Desde entonces hasta la fecha, diversidad de estudios han acumulado un importante cuerpo de evidencia, de manera tal que para la psicología contemporánea la base universal de la expresión emocional es considerada como un aspecto pancultural del funcionamiento psicológico (Sauter et al., 2010).

A pesar de la exhaustiva investigación sobre reconocimiento de emociones básicas en los últimos años, la literatura sobre el tema revela ciertas inconsistencias con respecto a la relación entre el reconocimiento de expresiones faciales y el desarrollo (van Beek \& Dubas, 2008). Si bien los estudios sugieren que no es hasta que los sistemas de percepción maduran, alrededor de los cinco años, que los niños adquieren la capacidad para reconocer la mayoría de las expresiones emocionales frecuentes (Thomas et al., 2007), hasta la fecha son escasas las investigaciones que hayan examinado la trayectoria evolutiva del reconocimiento de las emociones faciales durante la infancia y la adolescencia. La mayoría de los estudios centran su atención en el período de la primera infancia, comprendida desde el nacimiento hasta los cinco años (McClure, 2000), con pocos desarrollos en edades comprendidas entre los seis y dieciocho años de edad (Gao \& Maurer, 2010; Mancini et al., 2013; Ross et al., 2012; Szekely, 2013). A su vez, la mayoría de los estudios están centrados en población con patología (Bilgi et al., 2017; English et al., 2018;), existiendo escasos trabajos sobre población típica (Chaplin \& Aldao, 2013). 
El reconocimiento y la expresión de emociones faciales resultan de gran importancia en el niño, ya que son el canal a través del cual son capaces de transmitir sus necesidades (Del Barrio, 2005). Las investigaciones al respecto, sugieren que los niños manifiestan una serie de rasgos característicos en el modo de procesar la información emocional: (1) responden con similar intensidad a todas las emociones; (2) presentan una alta frecuencia de aparición y transitoriedad emocional, pasando con facilidad de una emoción a otra; (3) el efecto del aprendizaje en las reacciones emocionales se empieza a observar durante el crecimiento, y con el paso del tiempo, el reconocimiento de algunas emociones se debilita mientras otras se fortalecen (Molina et al., 2010). Estas características aún no han sido lo suficientemente contrastadas como para arribar a conclusiones determinantes.

Por otro lado, teniendo en cuenta que la precisión del reconocimiento, incluso en niños pequeños, es relativamente buena, una cuestión que ha de abordarse es si hay cambios cualitativos en el desarrollo del reconocimiento de expresiones faciales durante la infancia y la adolescencia $y$, en caso afirmativo, si estos cambios pueden influir en la precisión del reconocimiento.

Los hallazgos sugieren que el desarrollo de esta habilidad representa un progreso perfeccionado durante la infancia y la adolescencia, en gran parte dado al desarrollo de ciertas regiones neuronales con directa injerencia en el reconocimiento emocional (Rodger et al., 2015). Los hallazgos neuro-estructurales muestran que las regiones del cerebro implicadas en la generación y regulación de las emociones continúan desarrollándose durante la adolescencia y más allá, y que la misma puede representar un momento de especial plasticidad para funciones que yacen sobre estos circuitos (Mills et al., 2014). También muestran que el desarrollo estructural no siempre se produce de forma lineal dentro de las áreas del cerebro (Mills et al., 2014; Shaw et al., 2008), ni se produce de manera uniforme a través de las múltiples regiones del cerebro. A su vez, se observa que las diferentes regiones del cerebro involucradas en el procesamiento de las emociones se desarrollan a ritmos diferentes en el mismo individuo, con las conectividades entre estas regiones también en proceso de cambio. Se ha sugerido que esto puede tener consecuencias funcionales, en particular para el procesamiento y el comportamiento socioemocional durante la adolescencia, cuando se produce la mayor parte de este desarrollo.

Basados en estas evidencias estructurales y funcionales del cerebro, De Sonneville et al. (2002) especulan que el reconocimiento de expresiones faciales emocionales no emerge en una etapa específica del desarrollo, como una habilidad adquirida de una vez y para siempre, sino mediante un proceso graduado y perfectible a lo largo del tiempo. Son pocas las investigaciones que se propusieron evaluar el desarrollo particular del reconocimiento de cada modalidad emocional. Estas concluyen en que las habilidades emergen gradualmente con el tiempo, siendo la alegría reconocida más tempranamente y con la mayor exactitud, seguida de expresiones de tristeza y enojo. Las expresiones de sorpresa, miedo y asco son reconocidas más tardíamente (Gao \& Maurer, 2010; Gordillo, 2015; Lawrence et al., 2015; Ross et al., 2012). El siguiente trabajo se propone, por tanto, caracterizar el reconocimiento de emociones básicas en función de la edad en población infanto-juvenil.

\section{MÉTODO}

\section{Participantes}

La muestra está compuesta por 147 personas de ambos sexos, de entre 9 y 18 años. Del total de la muestra un $53,1 \%$ eran mujeres $(\mathrm{N}=78)$ y un $46,9 \%$ eran varones $(\mathrm{N}=69)$. Los participantes se seleccionaron de instituciones educativas (primarias y secundarias) de la ciudad de Mar del Plata. La participación fue voluntaria y sujeta al consentimiento de los padres y el asentimiento personal de los niños y adolescentes. Para la implementación de la presente investigación se consideraron los procedimientos recomendados por la American Psychological Association, los principios establecidos por la Convención Internacional sobre los Derechos del Niño y la Declaración de Helsinki. Asimismo, se respetaron los principios éticos de la investigación con seres humanos, procurándose las condiciones necesarias para proteger la confidencialidad y actuar en beneficio de los participantes. Se excluyeron individuos que presentaban trastornos del desarrollo neurológico (según criterios del DSM-V) y, por tratarse de pruebas con estímulos visuales, a aquellos participantes con dificultades visuales severas.

\section{Instrumentos de Evaluación}

Para la evaluación del reconocimiento de emociones básicas se empleó una tarea ampliamente utilizada en estudios transculturales e investigación neuropsicológica, el Test Pictures of Facial Affects (POFA; Ekman \& Friesen, 1969). El mismo utiliza los rostros faciales como estímulos para el reconocimiento de la información emocional, siendo el método más empleado hasta la actualidad (Anguas-Wong $\&$ Matsumoto, 2007). El POFA consta de 110 fotografías (en formato digital .TIF) y cuenta con estudios de sensibilidad y especificidad en población argentina (Tabernero \& Politis, 2012; Vaiman et al., 2011). Las 110 fotografías (en formato .TIF) que componen el set, muestran rostros completos en blanco y negro de 10 personas (6 mujeres, 4 hombres) que representan las seis emociones básicas: alegría, miedo, enojo, tristeza, sorpresa y asco, y expresiones neutras. 
Para su administración las imágenes se presentaron secuencialmente y en forma aleatoria, intercalando las seis emociones básicas y las expresiones neutras en la pantalla de un ordenador. Las fotografías tenían una dimensión de $7 \times 10 \mathrm{~cm}$ y se presentaban en el centro de la pantalla. Para la puntuación se le asignó un punto a cada acierto y cero puntos a cada error, constituyéndose una escala de valor mínimo 0 y valor máximo 110 . A partir de la digitalización se pudo agregar, a la prueba original, la medición de los tiempos de reacción empleados para el reconocimiento de cada emoción. Los tiempos de reacción se midieron a través del registro exacto del tiempo transcurrido entre la aplicación de un estímulo y el principio de la respuesta del sujeto a quien le fue presentado el estímulo. Una vez que el participante comenzaba a mencionar la emoción, el administrador, haciendo clic en la pantalla, detenía el tiempo de reacción. Con esta acción se abría una ventana emergente que presentaba las siete etiquetas verbales para su selección. Los nombres de estas seis emociones no eran visibles en la pantalla de la computadora, sólo aparecían una vez que el sujeto daba su respuesta. El tiempo comprendido para seleccionar el botón con la etiqueta correcta, no era tenido en cuenta en el registro del tiempo de reacción. El tiempo límite de presentación era de 5 seg., culminados estos, el programa pasaba automáticamente hacia el siguiente estímulo.

\section{Procedimiento}

En un primer momento se seleccionaron los participantes de dos instituciones educativas privadas (primarias y secundarias). A través de las instituciones escolares se estableció relación con familias de niños y adolescentes que asistían a estas escuelas y se les explicaron las características y objetivos de la investigación. Aclarando que la colaboración era voluntaria y anónima, se les solicitó la firma de un consentimiento informado a los adultos responsables y un asentimiento de los niños y adolescentes participantes. Luego, se administró el instrumento propuesto en un encuentro individual de aproximadamente 20 minutos, a aquellos estudiantes que decidieron participar, previo consentimiento y asentimiento informado. La presentación se realizó mediante una computadora portátil de 14 pulgadas en una habitación tranquila y adecuadamente iluminada. En caso de que los niños utilizaran anteojos, se les solicitó que realizaran la prueba con ellos puestos. La computadora se encontraba a una distancia aproximada de $60 \mathrm{~cm}$ del participante, el cual se posicionaba en dirección central al monitor, mientras que el experimentador se ubicaba a su derecha, puesto que era él quien utilizaba los comandos de la computadora ante las respuestas del participante.

\section{Análisis de Datos}

Se realizaron análisis de frecuencia y análisis descriptivos univariados y bivariados. Para contrastar las hipótesis de diferencias en el reconocimiento de emociones básicas se utilizaron estadísticos inferenciales, previamente al armado de categorías. Para analizar si los datos se ajustaban a la distribución normal se aplicó la prueba de KolmogorovSmirnov, dando como resultado que todas las variables referidas a la proporción de aciertos para cada emoción no se ajustan al modelo normal $(\mathrm{p}<, 001)$. Por este motivo, se optó por la utilización de pruebas de asociación no paramétricas, particularmente el coeficiente Rho de Spearman. Las variables referidas a tiempo de reacción en aciertos por cada tipo de emoción se ajustan al modelo normal (p>,001), lo cual permitió utilizar pruebas paramétricas. En este caso se optó por un modelo de regresión lineal en el cual se consideró como variable dependiente al tiempo de respuesta en cada emoción, como co-variable a la edad y como factor al sexo. Se utilizó un nivel de significación de $\alpha=, 05$. Previamente a realizar el análisis sobre los tiempos de respuesta, se eliminaron aquellas puntuaciones que representaban dos desvíos estándar por encima y por debajo de la media.

\section{RESULTADOS}

En la Figura 1 se pueden ver los gráficos de dispersión correspondientes a la relación entre la edad y los porcentajes de aciertos para cada emoción.

En la Tabla 1 se exponen los resultados del coeficiente de correlación Rho de Spearman entre la variable edad y las variables de aciertos en las distintas emociones.

La asociación entre edad y tasa de aciertos arrojó correlaciones significativas negativas en el reconocimiento de la alegría $\left(r_{\mathrm{s}}=-, 253, p .=, 002\right)$. Por otro lado, se hallaron correlaciones significativas positivas entre edad y aciertos en $\operatorname{asco}\left(r_{\mathrm{s}}=, 221, p .=, 009\right)$ y miedo $\left(r_{\mathrm{s}}=, 212, p .=, 011\right)$.

En la Figura 2 se exponen los gráficos de dispersión de la relación entre la edad y los tiempos de reacción por cada tipo de emoción.
En la Tabla 2 se exponen los resultados del modelo lineal general realizado sobre las variables referidas a tiempos de reacción para cada emoción. Dado que hay estudios que indican que el sexo también influye en los tiempos de reacción del reconocimiento emocional (Lawrence et al., 2015; López Morales et al., 2017; Torro-Alves et al., 2013) se decidió incluirlo como factor en el análisis.

Se puede observar un efecto significativo de la edad sobre todas las variables, a excepción de miedo. En el caso de alegría, enojo y sorpresa el tamaño del efecto fue moderado, mientras que en asco y tristeza el tamaño del efecto fue débil. Esta misma tendencia se vio reflejada en la potencia. Como se puede ver en la Figura 2, a mayor edad disminuyen los tiempos de respuesta para estas variables. 

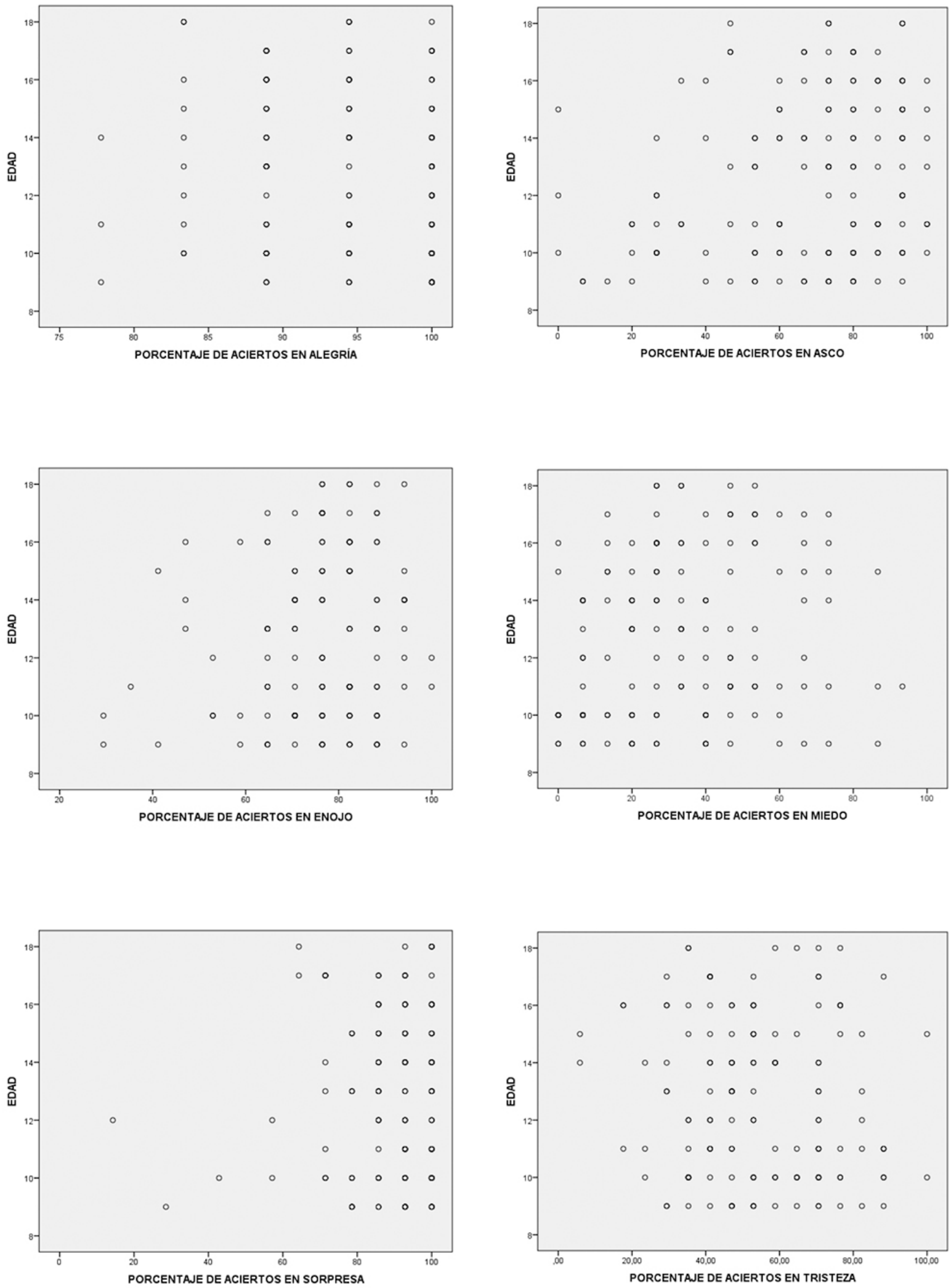

Figura 1. Gráficos de dispersión correspondientes a la relación entre la edad y los porcentajes de aciertos para cada emoción

Por otro lado, se observa un efecto significativo del sexo sobre las variables alegría, asco, sorpresa y tristeza, aunque el tamaño del efecto es menor que el de la edad. En el caso del miedo, donde no se observaba efecto de la edad, sí aparece un efecto del sexo. Como se puede observar en la Tabla 2, las mujeres tuvieron mayor porcentaje de aciertos en las cuatro primeras variables y los hombres en miedo. 

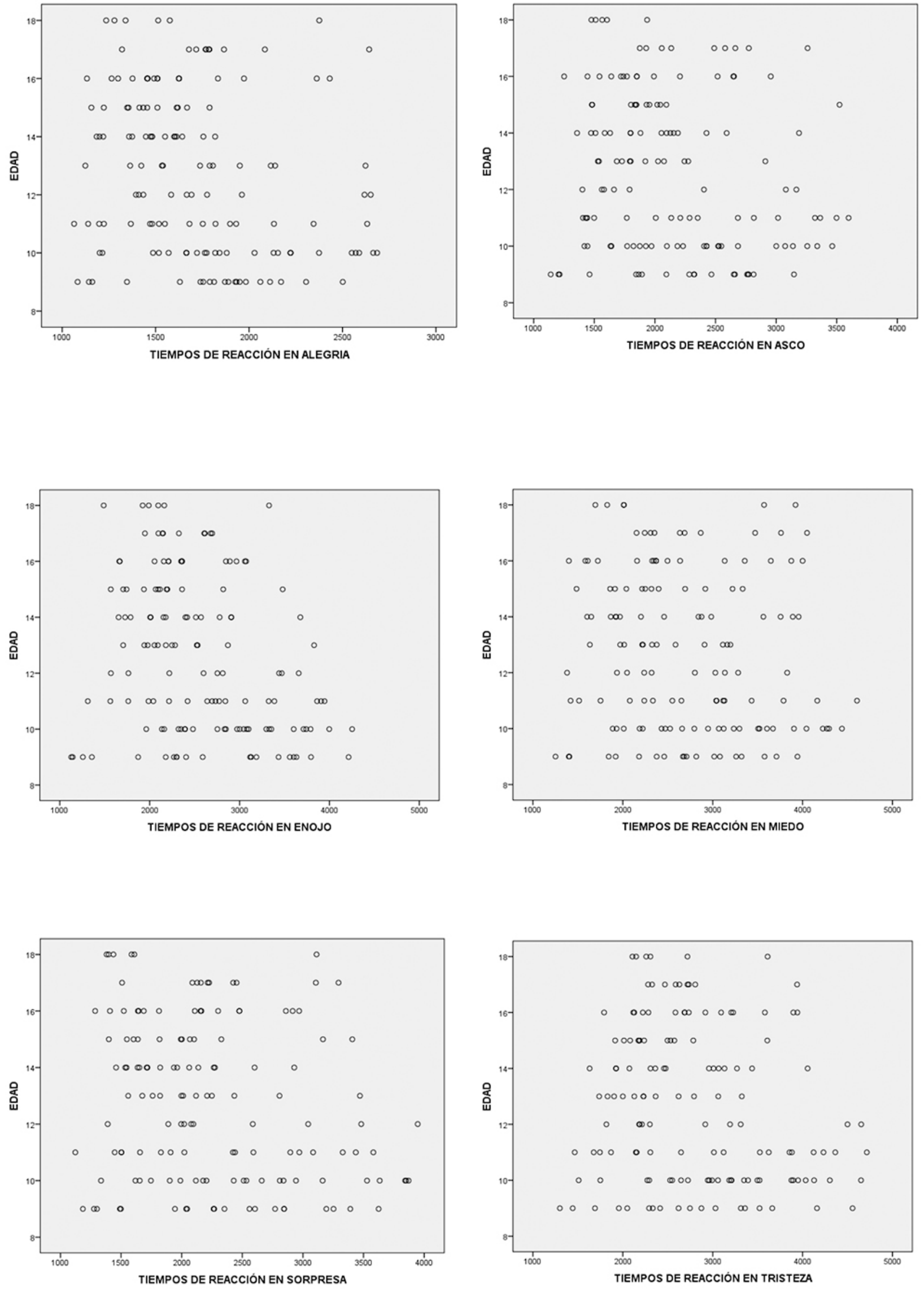

Figura 2. Gráficos de dispersión correspondientes a la relación entre la edad y los tiempos de reacción para cada emoción. 
Tabla 1

Resultados del Coeficiente de Correlación Rho de Spearman entre la variable edad y las variables de aciertos en las distintas emociones

\begin{tabular}{|c|c|c|c|c|c|c|c|}
\hline & & Alegría & Asco & Enojo & Miedo & Sorpresa & Tristeza \\
\hline \multirow[t]{3}{*}{ Edad } & Coeficiente de correlación &,$- 253^{* *}$ &, $221^{* *}$ &, 161 &, $210^{*}$ &, 007 &,- 094 \\
\hline & Sig. (bilateral) & ,002 & ,009 & ,062 & 011 & ,930 & ,265 \\
\hline & $\mathrm{N}$ & 143 & 139 & 135 & 147 & 141 & 142 \\
\hline
\end{tabular}

* La correlación es significativa al nivel 0,05 (bilateral).

** La correlación es significativa al nivel 0,01 (bilateral).

Tabla 2

Modelo Lineal General realizado sobre los Tiempos de Reacción para cada emoción

\begin{tabular}{lcccccc}
\hline TR por emoción & Origen & $\boldsymbol{G l}$ & $\boldsymbol{F}$ & \multicolumn{1}{c}{ Sig. } & ETA parcial al cuadrado & Potencia observada \\
\hline \multirow{2}{*}{ Alegría $^{\mathrm{a}}$} & Edad & 1 & 8,234 &, 005 &, 056 &, 813 \\
& Sexo & 1 & 6,196 &, 014 &, 042 &, 696 \\
\multirow{2}{*}{ Asco $^{\mathrm{b}}$} & Edad & 1 & 4,493 &, 036 &, 032 &, 558 \\
& Sexo & 1 & 4,030 &, 047 &, 029 &, 513 \\
Enojo $^{c}$ & Edad & 1 & 12,464 &, 001 &, 083 &, 939 \\
& Sexo & 1 & 2,023 &, 157 &, 015 &, 292 \\
Miedo $^{d}$ & Edad & 1 & 2,356 &, 127 &, 017 &, 332 \\
& Sexo & 1 & 3,905 &, 050 &, 028 &, 501 \\
Sorpresa $^{\mathrm{e}}$ & Edad & 1 & 7,398 &, 007 &, 051 &, 771 \\
& Sexo & 1 & 6,531 &, 012 &, 045 &, 718 \\
Tristeza $^{\mathrm{f}}$ & Edad & 1 & 4,105 &, 045 &, 029 &, 521 \\
\hline
\end{tabular}

$\mathrm{R}$ al Cuadrado $=, 099(\mathrm{R}$ al Cuadrado Ajustada $=, 087)$.

$\mathrm{R}$ al Cuadrado $=, 064(\mathrm{R}$ al Cuadrado Ajustada $=, 050)$.

$\mathrm{R}$ al Cuadrado $=, 101(\mathrm{R}$ al Cuadrado Ajustada $=, 088)$.

$\mathrm{R}$ al Cuadrado $=, 046(\mathrm{R}$ al Cuadrado Ajustada $=, 032)$.

$\mathrm{R}$ al Cuadrado $=, 096(\mathrm{R}$ al Cuadrado Ajustada $=, 083)$.

$\mathrm{R}$ al Cuadrado $=, 046(\mathrm{R}$ al Cuadrado Ajustada $=, 032)$.

\section{DISCUSIÓN}

El reconocimiento de emociones a través de la información brindada por los rostros es de cabal importancia en los procesos de interacción social humana. Los rostros no sólo proporcionan información visual que permite determinar el sexo, la edad, la familiaridad y la identidad de un individuo, también se utiliza la configuración de las caras para reunir información acerca de lo que otras personas podrían estar pensando o sintiendo (He \& Johnson, 2018; van den Boomen et al., 2017). El análisis y la interpretación de las expresiones faciales de la emoción son necesarios para modular las interacciones sociales humanas de manera apropiada. Este trabajo se propuso caracterizar el reconocimiento de emociones básicas en función de la edad en población infanto-juvenil

En lo que respecta a la tasa de aciertos, en primer lugar, se halló una asociación significativa negativa entre la edad y el reconocimiento de la alegría (Tabla 1). Si bien en la revisión de la literatura no se encontró este mismo resultado, algunos estudios observaron una ausencia de mejoría en el reconocimiento de esta emoción a lo largo del desarrollo, contrastante con la mejoría en otras emociones. Mancini y colaboradores (2013) observaron un aumento en la precisión del reconocimiento durante el período de 8-12 años para todas las emociones, excepto para rostros alegres, donde no se hallaron diferencias. En la misma línea, Rodger y colaboradores (2015) encontraron que la sensibilidad a las expresiones emocionales aumentó desde los cinco años de edad hasta la edad adulta, para todas las expresiones, excepto las de la alegría y el miedo. Según esos estudios los niños pequeños poseen niveles iguales de sensibilidad comparados a la de los adultos para la detección de la alegría y el miedo. Es importante atender a las diferencias metodológicas entre ambos estudios dado que el presente trabajo utilizó el etiquetado de emociones, mientras que el estudio de Rodger et al. (2015) evaluaba los umbrales perceptuales. Los resultados de este último sugieren que a los seis años de edad se alcanza el nivel de desarrollo perceptivo necesario para identificar la alegría, pero aún no 
para el resto de las emociones (o al menos con la precisión de un adulto). Nuestro estudio se ubica en una edad algo posterior (a partir de los nueve años) donde suponemos que el reconocimiento de esta emoción ya está consolidado. La prueba utilizada propone asociar el rostro con la etiqueta verbal, lo cual requiere un procesamiento más complejo. Un intento de explicar nuestros resultados, aún en contra de lo encontrado por otros autores es referir al alto valor adaptativo que tienen las expresiones emocionales durante la niñez. Las expresiones de alegría e interés indican a los niños que la otra persona tiene buena disposición para mantener la relación, lo que motiva a prolongar la interacción (Ortíz et al., 1999). La transmisión y reconocimiento de emociones entre el niño y los adultos significativos son primordiales para generar un vínculo afectivo, esencial en la comunicación, pues las reacciones emocionales del niño ayudan al adulto a entender sus necesidades básicas durante la infancia, donde mayor dependencia tiene el sujeto humano (Susskind et al., 2008). De esta forma, al interpretar correctamente las expresiones emocionales alegres, el niño puede regular su conducta en función de una comunicación adecuada y de la interacción social positiva (Fiske \& Taylor, 2013). Asimismo, las emociones positivas, en este caso la alegría, tienen una función especial para la supervivencia de la especie, facilitando la comunicación e interacción social, como así también la adaptación al medio ambiente, basadas en la empatía y en la asertividad social (Susskind et al., 2008; Vaiman et al., 2011).

En segundo lugar, se encontró una asociación significativa positiva entre la edad y el reconocimiento del asco y el miedo. Este resultado es consistente con lo reportado por Mancini y colaboradores (2013), Rodger y colaboradores (2015) y Lawrence y colaboradores (2015). Para intentar explicar estos resultados se pueden tener en cuenta las observaciones realizadas por Scherf, Behrmann y Dahl (2012) que en una revisión muy detallada relativa a la interacción entre el reconocimiento de rostros, la adolescencia y el desarrollo del comportamiento, sugieren que, como la adolescencia está representada por un intento de alejarse de sus padres, y reorientarse hacia sus compañeros, hay un aumento del deseo de aceptación de su endogrupo y el aumento de la sensibilidad a la evaluación entre iguales. Esto puede dar lugar a cambios cualitativos en el tipo de información que se extrae de las caras, con una mayor sensibilidad a las muestras de valoración negativa por los pares. Las expresiones de asco podrían concebirse como una señalización de desaprobación y juicios negativos hacia el receptor. En este sentido, el hallazgo parece ser consistente con la observación de que, cuando los niños entran en la adolescencia, están cada vez más obligados a buscar la aceptación social de sus pares, y se vuelven extremadamente sensibles a la evaluación entre iguales (Scherf et al., 2012; Steinberg \& Morris, 2001).

En lo que respecta al reconocimiento del miedo y el asco nuestros resultados parecen ser consistentes con la teoría propuesta por Widen (2013), quien ha sugerido que los niños tienden a dividir las expresiones faciales emocionales en dos categorías (se siente bien o se siente mal) y sólo poco a poco este sistema de clasificación se someta a cambios cualitativos, lo que permite a los niños utilizar cada vez más categorías específicas y diferenciadas. Dentro de la categoría se siente bien, se incluye solo a la alegría, mientras que se siente mal incluye miedo, enojo, asco y tristeza, lo que explicaría el contraste entre el reconociendo temprano adecuado de la alegría en contraste con las emociones negativas. De acuerdo con esta teoría, la distinción posterior realizada dentro de las categorías se siente mal o se siente bien es un proceso gradual y perfectible a lo largo de la adolescencia.

La línea de investigación en reconocimiento emocional y desarrollo arroja una variedad considerable de resultados, en muchos casos contrapuestos, y en otros con algunas coincidencias empíricas. Lo que sí parece tener acuerdo conceptual es el hecho del perfeccionamiento a lo largo del tiempo en la velocidad para reconocer las emociones básicas faciales. El presente estudio halló descenso en las medias de tiempos de reacción para el reconocimiento de todas las emociones básicas, a excepción del miedo (Tabla 2). Esto denota mayores aptitudes en el reconocimiento emocional en adolescentes, lo que condice con algunos estudios precedentes (Lawrence et al., 2015; Mancini et al., 2013; Rodger et al., 2015). Los estudios de imágenes del cerebro indican que las redes del cerebro implicadas en el reconocimiento de emociones no se comparan a la de los adultos hasta el final de la infancia o la adolescencia temprana (Ross et al., 2012). Estos estudios sugieren la no linealidad en el desarrollo de esta función social, con un aumento constante en el rendimiento o la actividad cerebral especializada desde la infancia hasta el comienzo de la adolescencia, seguido de pequeños cambios en el reconocimiento de emociones durante este período, culminando la optimización del proceso con mejoras al finalizar la adolescencia hasta la edad adulta. Con respecto a la ausencia de efecto de la edad en los tiempos de reacción en el reconocimiento del miedo, cabe observar que esta emoción fue la que presentó mayores tasas de error en general. Con lo cual, es factible que, aunque aumente el nivel de aciertos con la edad, su identificación siga siendo lenta, aún para los adolescentes.

A su vez, el desarrollo de las relaciones funcionales corticales y subcorticales prefrontales, en esta etapa evolutiva, podría aportar soporte teórico para pensar el desarrollo en el reconocimiento de emociones faciales. Dentro de los primeros 12 años de vida, hay un gran aumento de la mielinización, lo que mejora la eficiencia entre las conexiones corticales y subcorticales (Herschkowitz, 2000). A su vez, los axones que constituyen estas vías de conexión continúan desarrollándose en la adolescencia (Paus et al., 1999). Las mejoras relacionadas con la edad en el reconocimiento de expresiones emocionales podrían depender del perfeccionamiento de las conexiones 
estructurales y funcionales entre estructuras corticales (Dumontheil, 2014). Lo expuesto podría ser un argumento sólido para justificar el perfeccionamiento progresivo del reconocimiento emocional, con bases neuroanatómicas y consecuente perfeccionamiento en el procesamiento de información social.

Desde otro punto de vista, algunos autores sugieren, que la acumulación de nuevas experiencias sociales desde la niñez a la adolescencia como, por ejemplo, el ingreso a la escuela secundaria o la participación en múltiples tipos de grupalidades (fiestas, salidas, viajes, equipos deportivos) pueden influir en el desarrollo de los procesos cognitivos sociales, haciendo más óptimas las habilidades interpersonales, entre ellas, el reconocimiento facial emocional (Blakemore \& Choudhury, 2006).

Cabe mencionar que hay otras variables implicadas en la capacidad para reconocer emociones a través de rostros. En este trabajo incluimos al sexo, el cual ha reportado tener un efecto en esta función (Lawrence et al., 2015; López Morales et al., 2017). Se observó que las mujeres presentaban menores tiempos de reacción en el reconocimiento de alegría, asco y sorpresa, mientras que los hombres los tenían en miedo. Sin embargo, el tamaño del efecto fue débil. Los resultados han sido reportados con mayor detalle en un trabajo previo (López Morales et al., 2017).

Los datos recabados apoyan la importancia de seguir en esta línea de investigación con el fin de comprender cómo se produce el desarrollo normal en la capacidad para reconocer las emociones, ya que podría ser de utilidad a la hora de identificar posibles problemas como los trastornos del espectro autista, la depresión y los trastornos de ansiedad en esta población. Además de potenciar competencias emocionales, que redunden en la resiliencia para mejorar la calidad de vida de niños y adolescentes, en tanto que las emociones son un componente necesario de la interacción social, que permiten la transmisión de la información de manera eficaz.
En cuanto a las limitaciones del estudio, es relevante destacar el posible sesgo que podría representar que se utilicen instrumentos con expresiones faciales en rostros de adultos para analizar el desempeño de población infantojuvenil, como ya se ha demostrado en el reconocimiento facial en un estudio realizado por Proietti et al. (2014). Es decir, los resultados actuales pueden subestimar la verdadera capacidad de los niños y adolescentes para reconocer ciertas expresiones emocionales en rostros. Queda pendiente indagar, en estudios posteriores, si esas mismas expresiones, observadas en rostros de personas cercanas a su edad, mejoran el desempeño de esta población. Sería interesante indagar, a su vez, los procesos implicados en el reconocimiento de emociones a través de la información brindada por el cuerpo o la mirada, y observar si existen relaciones con el reconocimiento de expresiones faciales emocionales y si el desarrollo actúa de modo diferencial en estas variables. Por su parte, a futuro sería relevante ampliar los rangos de edad, para observar si las diferencias halladas en este estudio se mantienen, varían o, incluso, si el reconocimiento de otras emociones que no demostraron diferencias significativas en este estudio comprueban ser diferentes en función de la etapa del desarrollo.

Por otro lado, una limitación del estudio está representada por el modo de registrar los tiempos de reacción en el reconocimiento emocional. Como la latencia de la respuesta fue registrada mediante una acción motora del experimentador en la pantalla de la computadora, los registros podrían ser levemente imprecisos, viéndose alterados por las capacidades sensoriales del experimentador en la detección de la voz y la ejecución posterior de una respuesta motora para detener el registro de los tiempos de reacción. Diferencias sutiles de milisegundos podría proporcionar datos menos precisos que podrían limitar el alcance de las conclusiones. Para estudios posteriores, se podría atenuar estos afectos mediante softwares de registro de la respuesta vocal (voice key).

\section{REFERENCIAS}

Adolphs, R. (2002). Recognizing emotion from facial expressions: Psychological and neurological mechanisms. Behavioral Cognitive Neuroscience Reviews, 1(1), 21-62. https://doi.org/ $10.1177 / 1534582302001001003$

Adolph, R. (2010). Conceptual challenges and directions for Social Neuroscience. Neuron, 65(6), 752-767. https://doi. org/10.1016/j.neuron.2010.03.006

Anguas-Wong, A. M., \& Matsumoto, D. (2007). Reconocimiento de la expresión facial de la emoción en mexicanos universitarios. Revista de Psicología, 15(2), 277-293. http://www.redalyc.org/ articulo.oa? $\mathrm{id}=337829542006$

Bilgi, M.M., Taspinar, S., Aksoy, B., Oguz, K., Coburn, K., \& Gonul, A.S. (2017). The relationship between childhood trauma, emotion recognition, and irritability in schizophrenia patients. Psychiatry Research, 251, 90-96. https://doi.org/10.1016/j. psychres.2017.01.091

Blakemore, S. J., \& Choudhury, S. (2006). Development of the adolescent brain: Implications for executive function and social cognition. Journal of Child Psychology and Psychiatry, 47(3), 296-312. https://doi.org/10.1111/j.14697610.2006.01611.x

Chaplin, T. M., \& Aldao, A. (2013). Gender differences in emotion expression in children: A meta-analytic review. Psychological Bulletin, 139(4), 735-65. https://doi.org/10.1037/a0030737

Damasio, A.R. (2005). En busca de Spinoza: Neurobiología de la emoción y los sentimientos. Editorial Crítica.

De Sonneville, L.M.J., Verschoor, C.A., Njiokiktjien, C., Op het Veld, V., Toorenaar, N., \& Vranken, M. (2002). Facial identity and facial emotions: Speed, accuracy, and processing strategies in children and adults. Journal of Clinical and Experimental Neuropsychology, 24, 200-213. https://doi.org/10.1076/ jcen.24.2.200.989

Del Barrio, M. V. (2005). Emociones infantiles. Evolución, evaluación y prevención. Pirámides.

Dumontheil, I. (2014). Development of abstract thinking during childhood and adolescence: The role of rostrolateral prefrontal 
cortex. Developmental Cognitive Neurosciences, 10, 57-76. https://doi.org/10.1016/j.den.2014.07.009

Dunbar, R. I. (2009). The social brain hypothesis and its implications for social evolution. Annals of Human Biology, 36(5), 562-572. https://doi.org/10.1080/03014460902960289

Ekman, P. (1972). Universals and cultural differences in facial expressions of emotion. In J. Cole (Ed.), Nebraska Symposium on Motivation, 1971 (pp. 207-283). University of Nebraska Press. https://www.paulekman.com/wp-content/ uploads/2013/07/Universals-And-Cultural-Differences-InFacial-Expressions-Of.pdf

Ekman, P. (1994). Strong evidence for universals in facial expressions: A reply to Russell's mistaken critique. Psychological Bulletin, 115, 268-287. https://doi.org/10.1037/0033-2909.115.2.268

Ekman, P. (1999). Basic Emotions. In T. Dalgleish \& M.J. Power (Eds.), Handbook of Cognition and Emotion (pp. 45-60). John Wiley and Sons.

Ekman. P., \& Friesen, W.V. (1969). The repertoire of nonverbal behavior: Categories, origins, usage, and coding. Semiotica, 1, 49-98. https://www.paulekman.com/wp-content/ uploads/2013/07/The-Repertoire-Of-Nonverbal-BehaviorCategories-Origins-.pdf

English, L. H., Wisener, M., \& Bailey, H. N. (2018). Childhood emotional maltreatment, anxiety, attachment, and mindfulness: Associations with facial emotion recognition. Child Abuse Neglect, 80, 146-160. https://doi.org/10.1016/j. chiabu.2018.02.006

Fiske, S. T., \& Taylor, S. K. (2013). Social Cognition: From Brains to Culture. SAGE.

Gao, X., \& Maurer, D. (2010). A happy story: developmental changes in children's sensitivity to facial expressions of varying intensities. Journal of Experimental Child Psychology, 107, 67-86. https://doi.org/10.1016/j.jecp.2010.05.003

García-Rodríguez, B., Fusari, A., \& Ellgring, H. (2008). Procesamiento emocional de las expresiones faciales en el envejecimiento normal y patológico. Revista Neurología, 46(10), 609-617. https://www.neurologia.com/articulo/2019268

Gordillo, F., Mestas, L., Salvador, J., Pérez, M-A., Arana, J. M., \& López, R. M. (2015). Diferencias en el reconocimiento de las emociones en niños de 6 a 11 años. Acta de Investigación Psicológica, 5(1), 1846-1859. https://doi.org/10.1016/S20074719(15)30005-3

Grande-García, I. (2009). Neurociencia Social: El maridaje entre la psicología social y las neurociencias cognitivas. Revisión e introducción a una nueva disciplina. Anales de Psicología, $25,1-20$.

He, W., \& Johnson, B. W. (2018). Development of face recognition: Dynamic causal modelling of MEG data. Developmental Cognitive Neuroscience, 30, 13-22. https://doi.org/10.1016/j. den.2017.11.010

Herschkowitz, N. (2000). Neurological bases of behavioural development in infancy. Brain and Development, 22, 411-416. https://doi.org/10.1055/s-2007-973720

Lawrence, K., Campbell, R., \& Skuse, D. (2015). Age, gender, and puberty influence the development of facial emotion recognition. Front Psychology, 6, 761. https://doi.org/10.3389/ fpsyg.2015.00761

Lieberman, M. D. (2010). Social cognitive neuroscience. In S. T. Fiske, D. T. Gilbert, \& G. Lindzey (Eds.), Handbook of Social Psychology (pp. 143-193). McGraw-Hill.

López Morales, H., Agulla, L., Zabaletta, V., Vivas, L., \& López, M. (2017). Rostros, gestos y emociones: Procesamiento diferencial de las expresiones faciales emocionales en población infantojuvenil según el sexo. Revista Argentina de Ciencias del Comportamiento, 9(3), 31-43. https://revistas.unc.edu.ar/index. $\mathrm{php} / \mathrm{racc} / \mathrm{article} / \mathrm{view} / 18521$
Mancini, G., Agnoli, S., Baldaro, B., Bitti, P.E., y Surcinelli, P. (2013). Facial expressions of emotions: recognition accuracy and affective reactions during late childhood. The Journal of Psychology 147, 599-617. https://doi.org/10.1080/00223980. 2012.727891

Matsumoto, D. (2001). The handbook of culture and psychology. Oxford University Press.

McClure, E. B. (2000). A meta-analytic review of sex differences in facial expression processing and their development in infants, children, and adolescents. Psychological Bulletin, 126(3), 424-453. http://www.ncbi.nlm.nih.gov/pubmed/10825784

Miguel, F. K. (2015). Psicologia das emoções: uma proposta integrativa para compreender a expressão emocional. Psico-USF, 20(1), 153-162. http://doi.org/10.1590/141382712015200114

Mills, K. L., Goddings, A. L., Clasen, L. S., Giedd, J. N., \& Blakemore, S. J. (2014). The Developmental mismatch in structural brain maturation during adolescence. Developmental Neuroscience, 36, 147-60. https://doi.org/10.1159/000362328

Molina, Y., Osses, C., Riquelme, C., Riquelme, V., Sepúlveda, S., \& Urrutia, A. (2010). Desarrollo social y emocional del párvulo. Universidad Católica de la Santísima concepción: Facultad de Educación.

Ortíz, M. J., Fuentes, M. J., \& López, F. (1999) Desarrollo socioafectivo en la primera infancia In J. Palacios, A, Marchesi, \& C. Coll. (Eds.) Desarrollo psicológico y educación. (pp. 151-175). Madrid: Alianza Editorial.

Paus, T., Zijdenbos, A., Worsley, K., Collins, D. L., Blumenthal, J., Giedd, J. N., Rapoport, J. L., \& Evans, A. C. (1999). Structural maturation of neural pathways in children and adolescents: In vivo study. Science, 283(5409), 1908-1911. http://www.ncbi. nlm.nih.gov/pubmed/10082463

Proietti, V., Macchi-Cassia, V., \& Mondloch, C.J. (2014). The ownage face recognition bias is task dependent. British Journal of Psychology, 106(3), 446-467. https://doi.org/10.1111/ bjop.12104

Rodger, H., Vizioli, L., Ouyang, X., \& Caldara, R. (2015). Mapping the development of facial expression recognition. Developmental Science 18(6), 926-39. https://doi.org/10.1111/ desc. 12281

Ross, P. D., Polson, L., \& Gosbras, M. H. (2012). Developmental changes in emotion recognition from full-light and point-light displays of body movement. PLOS ONE, 7(9), e44815. https:// doi.org/10.1371/journal.pone.0044815

Ruiz-Ruiz, J.C., García-Ferrer, S., \& Fuentes-Durá, I. (2006). La relevancia de la cognición social en la esquizofrenia. Apuntes de Psicología, 24(3),137-155. http://www.apuntesdepsicologia. es/index.php/revista/article/view/74

Russell, J. A. (1994). Is there universal recognition of emotion from facial expression? A review of the cross-cultural studies. Psychological Bulletin, 115, 102-141. http://www.ncbi.nlm. nih.gov/pubmed/8202574

Sauter, D. A., Eisner, F., Ekman, P., \& Scott, S. K. (2010). Crosscultural recognition of basic emotions through nonverbal emotional vocalizations. PNAS, 107, 2408-2412. https://doi. org/10.1073/pnas.0908239106

Scherf, K. S., Behrmann, M., \& Dahl, R. E. (2012). Facing changes and changing faces in adolescence: A new model for investigating adolescent-specific interactions between pubertal, brain and behavioral development. Developmental Cognitive Neurosciences, 2(2), 199-219. https://doi.org/10.1016/j. den.2011.07.016

Shaw, P., Kabani, N. J., Lerch, J. P., Eckstrand, K., Lenroot, R., Gogtay N., Greenstein D, Clasen, L., Evans, A., Rapoport, J. L., Giedd, J. N., \& Wise, S. P. (2008). Neurodevelopmental trajectories of the human cerebral cortex. Journal of 
Neuroscience, 28, 3586-3594. https://doi.org/10.1523/ jneurosci.5309-07.2008

Singer, T. (2012). The past, present and future of social neuroscience: A European perspective. Neuroimage, 61, 437-449. https://doi. org/10.1016/j.neuroimage.2012.01.109

Steinberg, L., \& Morris, A. (2001). Adolescent development. Annual Review of Psychology, 52(1), 83-110. https://doi.org/10.1146/ annurev.psych.52.1.83

Susskind, J. M., Lee, D.H., Cusi, A., Feiman, R., Grabski, W., \& Anderson, A.K. (2008). Expressing fear enhances sensory acquisition. Nature Neuroscience, 11(7), 843-850. https://doi. org/10.1038/nn.2138

Szekely, E. (2013). Children's emocional functioning in the preschool period: emotion recognition, temperament, and their links with early risk factors (Thesis). http://repub.eur. $\mathrm{nl} / \mathrm{pub} / 38600 /$

Tabernero, M. E., \& Politis, D. (2012). Evaluación del reconocimiento facial de emociones básicas en demencia frontotemporal variante frontal. Revista Argentina de Neuropsicología, 20, 24-34. https://doi.org/10.5579/rnl.2012.0127

Thomas, L. A., De Bellis, M.D., Graham, R., \& LaBar, K. S. (2007). Development of emotional facial recognition in late childhood and adolescence. Developmental Science, 10(5), 547-58. https://doi.org/10.1111/j.1467-7687.2007.00614.x
Torro-Alves, N., Oliveira-Bezerra, I. A., Gomes-Claudino, R., \& Laurindo-Pereira, T. C. (2013). Influences of sex, type and intensity of emotion in the recognition of static and dynamic facial expressions. Avances en Psicología Latinoamericana, 31(1), 192-199. http://www.scielo.org.co/scielo.php?script=sci_artt ext\&pid=S1794-47242013000100016

Vaiman, M., Caicedo, E., \& Pereno, G. (2011). La expresión de emociones en el Picture of Facial Affect: Índices de reconocimiento en una muestra de estudiantes universitarios. Revista Argentina de Neuropsicología, 19, 10-21. http:// www.scielo.org.mx/scielo.php?script $=$ sci_arttext\&pid $=\mathrm{S} 0185-33252013000200001$

van Beek, Y., y Dubas, J. S. (2008). Age and gender differences in decoding basic and non-basic facial expressions in late childhood and early adolescence. Journal of Nonverbal Behavior, 32(1), 37-52. doi: 10.1007/s10919-007-0040-8.

van den Boomen, C., Munsters, N. M., \& Kemner, C. (2017). Emotion processing in the infant brain: The importance of local information. Neuropsychologia, 17, 30332-30339. https://doi. org/10.1016/j.neuropsychologia.2017.09.006

Widen, S. C. (2013). Children's interpretation of facial expressions: the long path from valence-based to specific discrete categories. Emotional Review, 5(1), 72-77. https://doi. org/10.1177/1754073912451492 